\title{
On the difference property of Borel measurable functions
}

\author{
by
}

\author{
Hiroshi Fujita (Matsuyama) and Tamás Mátrai (Budapest)
}

\begin{abstract}
If an atomlessly measurable cardinal exists, then the class of Lebesgue measurable functions, the class of Borel functions, and the Baire classes of all orders have the difference property. This gives a consistent positive answer to Laczkovich's Problem 2 [Acta Math. Acad. Sci. Hungar. 35 (1980)]. We also give a complete positive answer to Laczkovich's Problem 3 concerning Borel functions with Baire- $\alpha$ differences.
\end{abstract}

1. Introduction. For each function $f: \mathbb{R} \rightarrow \mathbb{R}$ and each constant $h \in \mathbb{R}$, the difference function $x \mapsto f(x+h)-f(x)$ is denoted by $\Delta_{h} f$. If a class $\mathcal{F} \subseteq \mathbb{R} \mathbb{R}$ forms a translation invariant vector space over $\mathbb{R}$, then every $f \in \mathcal{F}$ satisfies the condition $\forall h \in \mathbb{R}\left[\Delta_{h} f \in \mathcal{F}\right]$.

If moreover $\mathcal{F}$ contains nonzero constant functions then every function of the form $f=g+\theta$, where $g$ is in $\mathcal{F}$ and $\theta$ is additive (i.e., $\theta(x+y)=\theta(x)+\theta(y)$ for every $x, y \in \mathbb{R}$ ), satisfies the same condition. The difference property is the converse of this trivial observation.

Definition 1.1. A class $\mathcal{F} \subseteq \mathbb{R} \mathbb{R}$ is said to have the difference property if every function $f: \mathbb{R} \rightarrow \mathbb{R}$ satisfying $\forall h \in \mathbb{R}\left[\Delta_{h} f \in \mathcal{F}\right]$ has the form $f=g+\theta$ where $g \in \mathcal{F}$ and $\theta$ is additive.

This notion was invented by N. G. de Bruijn, who proved that the class $C(\mathbb{R})$ of all continuous real functions has the difference property (see [2]). In fact, various subclasses of $C(\mathbb{R})$, which play important roles in classical analysis, have the difference property: for example, the class of differentiable functions, the class $C^{r}(\mathbb{R})$ of functions with continuous $r$ th derivatives, the class of real analytic functions, etc. These results are reviewed in M. Laczkovich's survey paper [14].

On the other hand, it was pointed out by P. Erdős that the second Baire class does not have the difference property if we assume the Continuum

2010 Mathematics Subject Classification: 03E15, 28A05, 54H15.

Key words and phrases: difference property, Lebesgue measurable functions, Borel functions, Baire class $\alpha$ functions, real-valued measurable cardinals. 
Hypothesis $(\mathrm{CH})$. Under $\mathrm{CH}$ there exists a set $A \subseteq \mathbb{R}$ such that for every $h \in \mathbb{R}$ the difference $(A+h) \backslash A$ is countable and yet $A$ is not Lebesgue measurable. The characteristic function of such a set $A$ has Baire-2 difference functions but it is not the sum of a Lebesgue measurable function and an additive function. Thus the difference property of the class of Lebesgue measurable functions or the class of Borel functions cannot be established under the conventional ZFC axioms of set theory.

The consistency of the difference property of the class $\mathcal{L}$ of Lebesgue measurable functions has been established by Laczkovich in [12] and [13]. In [12] Laczkovich proves that $\mathcal{L}$ has the weak difference property in the sense that every function $f$ satisfying $\forall h \in \mathbb{R}\left[\Delta_{h} f \in \mathcal{L}\right]$ is of the form $f=g+\theta+S$ where $g \in \mathcal{L}, \theta$ is additive and $S$ is small, i.e., $\Delta_{h} S(x)=0$ a.e. for every $h \in \mathbb{R}$. By this result, the consistency of the difference property of $\mathcal{L}$ is reduced to the problem of when every small function is Lebesgue measurable, which turns out to be a kind of strong Fubini theorem in which the measurability condition on the function of two variables is considerably relaxed. In [13], such a strong Fubini theorem is shown to be consistent with ZFC (see Section 5.1 for a precise statement). Along the same lines, in Section 3 we will prove the following theorem, which establishes the difference property of $\mathcal{L}$ assuming the existence of an atomlessly measurable cardinal.

THEOREM 1.2. Assume there exists an atomlessly measurable cardinal. Then the class of Lebesgue measurable functions has the difference property.

The above mentioned work of Laczkovich on Lebesgue measurable functions leaves open the consistency of the difference property of Borel functions. In [12], Laczkovich posed the following three problems motivated by the example of Erdös.

Problem 1. Does the first Baire class $\mathcal{B}_{1}$ have the difference property?

Problem 2. Suppose that $\Delta_{h} f$ is Borel for every $h \in \mathbb{R}$. Is there a countable ordinal $\alpha$ such that $\Delta_{h} f$ is Baire- $\alpha$ for all $h \in \mathbb{R}$ ?

Problem 3. Suppose that $f$ is Borel and $\Delta_{h} f$ is Baire- $\alpha$ for every $h \in \mathbb{R}$. Is then $f$ itself Baire- $\alpha$ ?

Problem 1 has been solved positively by Laczkovich himself. See [14, Section 7]. One finds there that some useful subclasses of $\mathcal{B}_{1}$ have the difference property: approximately continuous functions, derivatives, Darboux Baire-1 functions, etc.

A counter-example to Problem 2 has been given by R. Filipów and I. Recław assuming $\mathrm{CH}$ (see [5, Theorem 3.1]). Their use of $\mathrm{CH}$ is unavoidable, though it can be replaced by a version of the Covering Property Axioms (see [4, Corollary 5.1.11]). In Section 3 we will prove that the existence of an atomlessly measurable cardinal excludes such counter-examples; moreover, 
an atomlessly measurable cardinal implies that the class of Borel functions has the difference property.

THEOREM 1.3. Assume there exists an atomlessly measurable cardinal. Then the class of Borel functions has the difference property. Moreover, for every $\alpha<\omega_{1}$, the class of Baire- $\alpha$ functions has the difference property.

In Section 4, we will prove the following result, which provides a complete positive answer to Problem 3.

THEOREM 1.4. Let $f: \mathbb{R} \rightarrow \mathbb{R}$ be a Borel function and let $\alpha$ be a countable ordinal. Suppose that for every $h \in \mathbb{R}$ the difference function $\Delta_{h} f$ is of Baire class $\alpha$. Then $f$ itself is of Baire class $\alpha$.

Partial answers to Problem 3 were known earlier: Laczkovich [14, Section 7, p. 391] proved the statement for every bounded Borel function, while in [7] the first author solved the problem for every $\alpha \geq \omega$. Actually, it is the solution of Laczkovich for bounded functions which gives us the impression that the difference property of Borel functions should be consistent. However, our solution of Problem 3 goes in a quite different way from the approach of Laczkovich [14] or the first author [7]. We will use a Baire category argument while previous results used measure-theoretic methods. These three attempts are only loosely related to each other by the basic observation that questions about the difference property usually reduce to appropriate results on sections like e.g. the Baire-Namioka theorem, the Fubini theorem, the Kuratowski-Ulam theorem, etc.

We collect in Section 2 the preliminary results that we need. After obtaining our main results, in Section 5 we will discuss their possible generalizations under appropriate set-theoretic assumptions.

2. Preliminaries. The power set of $X$ is denoted by $\mathcal{P}(X)$. The cardinality of the continuum is denoted by $\mathfrak{c}$.

2.1. Sections of sets and functions. Let $X$ and $Y$ be sets and let $A \subseteq X \times Y$ be a set of pairs. The vertical section of $A$ at $x \in X$ is the set $A_{x}=\{y \in Y:\langle x, y\rangle \in A\}$. Similarly, the horizontal section of $A$ at $y \in Y$ is $A^{y}=\{x \in X:\langle x, y\rangle \in A\}$. For a function $F: X \times Y \rightarrow Z$ of two variables, we also define the vertical sections $F_{x}: Y \rightarrow Z(x \in X)$ and the horizontal sections $F^{y}: X \rightarrow Z(y \in Y)$ by $F_{x}(y)=F^{y}(x)=F(x, y)(x \in X, y \in Y)$.

2.2. Basic notions from descriptive set theory. Our reference for basic notions from descriptive set theory is [11. The $\alpha$ th additive (resp. multiplicative) class of the Borel hierarchy is denoted by $\boldsymbol{\Sigma}_{\alpha}^{0}$ (resp. $\boldsymbol{\Pi}_{\alpha}^{0}$ ). In particular, $\boldsymbol{\Sigma}_{1}^{0}$ denotes the class of open sets, $\boldsymbol{\Pi}_{1}^{0}$ the closed sets, $\boldsymbol{\Sigma}_{2}^{0}$ the $F_{\sigma}$ 
sets, etc. We define the $\alpha$ th ambiguous class by $\boldsymbol{\Delta}_{\alpha}^{0}=\boldsymbol{\Sigma}_{\alpha}^{0} \cap \boldsymbol{\Pi}_{\alpha}^{0}$. The class of all Borel sets is denoted by $\boldsymbol{B}$.

The $n$th additive (resp. multiplicative) class of the projective hierarchy is denoted by $\boldsymbol{\Sigma}_{n}^{1}$ (resp. $\boldsymbol{\Pi}_{n}^{1}$ ). Therefore $\boldsymbol{\Sigma}_{1}^{1}$ denotes the class of analytic sets, $\boldsymbol{\Pi}_{1}^{1}$ the coanalytic sets, etc. We define the $n$th ambiguous class by $\boldsymbol{\Delta}_{n}^{1}=\boldsymbol{\Sigma}_{n}^{1} \cap \boldsymbol{\Pi}_{n}^{1}$.

When we say a set $A$ is in one of the above defined pointclasses, we assume we know the ambient space of $A$. If we need to specify it, we write $\boldsymbol{\Sigma}_{\alpha}^{0}(X), \boldsymbol{\Pi}_{n}^{1}(Y)$, etc.

Let $X$ and $Y$ be Polish spaces and let $\boldsymbol{\Gamma}$ be a pointclass. We say a function $f: X \rightarrow Y$ is $\boldsymbol{\Gamma}$-measurable if for every open set $O \subseteq Y$ we have $f^{-1}[O] \in \boldsymbol{\Gamma}$. Therefore a function is $\boldsymbol{\Sigma}_{1}^{0}$-measurable if and only if it is continuous. A classical result, due to Lebesgue, says that a function from a Polish space $X$ to a Polish space $Y$ is of Baire class $\alpha$ if and only if it is $\boldsymbol{\Sigma}_{\alpha+1}^{0}$-measurable (see e.g. [11, (24.3) Theorem, p. 190]).

One can endow every $\boldsymbol{\Pi}_{2}^{0}$ subset of a Polish space with a complete metric without changing its topology; conversely, every completely metrizable subset of a metric space is $G_{\delta}$. For the details, we refer to [11, (3.11) Theorem, p. 17].

2.3. Universal measures. A measure space of the form $(Y, \mathcal{P}(Y), \mu)$ (i.e. where $\mu$ is defined for every subset of $Y$ ) is called a universal measure space.

It is fairly easy to construct on any set a universal probability measure space which is concentrated on a countable set of point masses (a point mass is a point with nonzero measure). On the other hand, the existence of a universal probability measure space without point masses is not provable in ZFC. The existence of such a universal measure is equivalent to the existence of a real-valued measurable cardinal, which is either two-valued measurable or atomlessly measurable.

Two-valued measurable cardinals are those cardinals that are usually called measurable cardinals in conventional terminology of set theory. They play a central role in the study of large cardinals.

An atomlessly measurable cardinal is an uncountable cardinal $\kappa$ carrying a universal probability measure space $(\kappa, \mathcal{P}(\kappa), \mu)$ which is $\kappa$-additive, i.e. the union of fewer than $\kappa \mu$-null sets is $\mu$-null, and atomless, i.e. whenever $\mu(A)>0$ then there is $B \subseteq A$ such that $0<\mu(B)<\mu(A)$. It is known that any atomlessly measurable cardinal is less than or equal to $\mathfrak{c}$. The abbreviation RVMC stands for the statement there exists an atomlessly measurable cardinal.

It is known that RVMC is consistent with ZFC if and only if so is the existence of a two-valued measurable cardinal. For more information about atomlessly measurable cardinals, including the equiconsistency result, we refer the reader to [6] and [19]. 
It is clear from the definition that the smallest cardinal $\kappa$ such that $\mu$ is not $\kappa$-additive is a successor cardinal. Thus we can define $\operatorname{add}(\mu)$ to be the largest cardinal $\kappa$ such that $\mu$ is $\kappa$-additive. In other words, $\operatorname{add}(\mu)$ is the smallest possible size of a family $\mathcal{A}$ of $\mu$-null sets such that $\bigcup \mathcal{A}$ is not $\mu$-null.

Lemma 2.1. Every $\sigma$-finite universal measure is $\omega_{2}$-additive.

Proof. Since $\mu$ is $\sigma$-finite, there is a partition $\left(Y_{n}\right)_{n<\omega}$ of $Y$ such that for every $n<\omega, \mu\left(Y_{n}\right)<\infty$. It is enough to verify that $\mu$ is $\omega_{2}$-additive on each $Y_{n}$ separately, so we can assume $Y=Y_{n}$ for some $n<\omega$. We consider only the nontrivial $\mu(Y)>0$ case.

Set $\kappa=\operatorname{add}(\mu)$. By definition, there is a family $\left(Y_{\alpha}\right)_{\alpha<\kappa}$ of pairwise disjoint subsets of $Y$ such that $\mu\left(Y_{\alpha}\right)=0$ for every $\alpha<\kappa$, but $\mu\left(\bigcup_{\alpha<\kappa} Y_{\alpha}\right)$ $>0$. Let $I=\left\{A \subseteq \kappa: \mu\left(\bigcup_{\alpha \in A} Y_{\alpha}\right)=0\right\}$. Then $I$ is a $\kappa$-complete $\sigma$-saturated ideal on $\kappa$. So by [9, Lemma 10.14, p. 132], $\kappa$ cannot be a successor cardinal, in particular $\kappa>\omega_{1}$.

2.4. Product $\sigma$-algebras. For a pair of $\sigma$-algebras $\mathcal{A}$ and $\mathcal{B}$ on sets $X$ and $Y$ respectively, let $\mathcal{A} \otimes \mathcal{B}$ be the $\sigma$-algebra on $X \times Y$ generated by $\{A \times B: A \in \mathcal{A}, B \in \mathcal{B}\}$. This is the smallest $\sigma$-algebra on $X \times Y$ that makes the projections $\pi_{X}: X \times Y \rightarrow X$ and $\pi_{Y}: X \times Y \rightarrow Y$ measurable. We call $\mathcal{A} \otimes \mathcal{B}$ the product $\sigma$-algebra of $\mathcal{A}$ and $\mathcal{B}$.

It is easy to see that for every $A \in \mathcal{A} \otimes \mathcal{B}$ and every $\langle x, y\rangle \in X \times Y$, the horizontal section $A^{y}$ and the vertical section $A_{x}$ are in $\mathcal{A}$ and $\mathcal{B}$ respectively. The converse is not true in general:

- The natural ordering relation $<$ on $\omega_{1}$, as a set of pairs, has the property that every horizontal section is countable and every vertical section is co-countable. So if $\mathcal{A}$ is the countable/co-countable algebra on $\omega_{1}$, then all horizontal and vertical sections of $<$ belong to $\mathcal{A}$. However, no ordering relation belongs to the product $\mathcal{A} \otimes \mathcal{A}$ because relations in $\mathcal{A} \otimes \mathcal{A}$ can never be anti-symmetric.

- If $\kappa>\mathfrak{c}$ then $\mathcal{P}(\kappa \times \kappa) \neq \mathcal{P}(\kappa) \otimes \mathcal{P}(\kappa)$ because the diagonal set does not belong to the right hand side.

However, as an important special case, the following is true.

Lemma 2.2. Let $X$ be a separable metric space and $Y$ be any nonempty set. Let $A \subseteq X \times Y$. Suppose that there is a countable ordinal $\alpha$ such that for every $y \in \bar{Y}$ the horizontal section $A^{y}$ is in $\boldsymbol{\Sigma}_{\alpha}^{0}(X)$. Then $A \in \boldsymbol{B}(X) \otimes \mathcal{P}(Y)$.

Proof. Let first $\alpha=1$. Let $\left\{N_{i}: i<\omega\right\}$ be an open basis in $X$. For every $i<\omega$, define $B_{i}=\left\{y \in Y: N_{i} \subseteq A^{y}\right\}$. Then it is routine to check that $A=\bigcup_{i \in \omega}\left(N_{i} \times B_{i}\right)$.

The $\alpha>1$ case is a straightforward induction on $\alpha$. 
Note that the converse of Lemma 2.2 is also true. Therefore the assumption $A^{y} \in \boldsymbol{\Sigma}_{\alpha}^{0}$ cannot be weakened to $A^{y} \in \boldsymbol{B}(X)$ unless $Y$ is countable.

2.5. The Fubini theorem. For a pair of $\sigma$-finite measure spaces, say $(X, \mathcal{A}, \lambda)$ and $(Y, \mathcal{B}, \mu)$, the product measure on $(X \times Y, \mathcal{A} \otimes \mathcal{B})$ will be denoted by $\lambda \otimes \mu$. We will need the following versions of the Fubini theorem (see e.g. [18, 8.8 Theorem, p. 164] or [17, Theorem 1 p. 325, Theorem 2 p. 329]).

Proposition 2.3 (Fubini theorem). Let $(X, \mathcal{A}, \lambda)$ and $(Y, \mathcal{B}, \mu)$ be $\sigma$ finite measure spaces. If a function $u: X \times Y \rightarrow \mathbb{R}$ is $\lambda \otimes \mu$-integrable then

(i) the vertical section function $u_{x}: y \mapsto u(x, y)$ is $\mu$-integrable for $\lambda$ almost every $x$,

(ii) the function $x \mapsto \int_{Y} u_{x}(y) d \mu(y)$ is $\mathcal{A}$-measurable,

(iii) $\iint_{X \times Y} u(x, y) d(\lambda \otimes \mu)(x, y)=\int_{X}\left(\int_{Y} u_{x}(y) d \mu(y)\right) d \lambda(x)$;

and similarly for the horizontal sections.

Proposition 2.4 (Fubini-Tonelli theorem). Let $(X, \mathcal{A}, \lambda)$ and $(Y, \mathcal{B}, \mu)$ be $\sigma$-finite measure spaces. Let $u: X \times Y \rightarrow \mathbb{R}$ be an $\mathcal{A} \otimes \mathcal{B}$-measurable function such that at least one of the iterated integrals

$$
\int_{X}\left(\int_{Y}\left|u_{x}(y)\right| d \mu(y)\right) d \lambda(x) \text { and } \int_{Y}\left(\int_{X}\left|u^{y}(x)\right| d \lambda(x)\right) d \mu(y)
$$

exists. Then $u$ is $\lambda \otimes \mu$-integrable and the conclusions of Proposition 2.3 follow.

For more on these results we refer the reader to textbooks on integrals, e.g., Halmos [8, Ch. VII], Taylor [20, Ch. 6] or Yeh [21, §23].

2.6. Category quantifiers. The $\sigma$-ideal of meager (i.e., of first category) sets in a topological space $X$ is denoted by $\mathcal{M}(X)$. We often write just $\mathcal{M}$ when the space $X$ is clear from the context.

Let $X$ and $Y$ be Polish spaces. Let $A \subseteq X \times Y$. We define two subsets $\exists^{* Y} A$ and $\forall^{* Y} A$ of $X$ by

$$
\begin{aligned}
& \exists^{* Y} A=\left\{x \in X: A_{x} \notin \mathcal{M}(Y)\right\}, \\
& \forall^{* Y} A=\left\{x \in X: Y \backslash A_{x} \in \mathcal{M}(Y)\right\} .
\end{aligned}
$$

In Section 4 we will need the following result due to Montgomery (see e.g. [11, (22.22) Exercise, p. 174]).

Lemma 2.5. Let $X$ and $Y$ be Polish spaces. Let $\alpha$ be a countable nonzero ordinal and let $B \subseteq X \times Y$ be a $\boldsymbol{\Sigma}_{\alpha}^{0}(X \times Y)$ set. Then $\exists^{* Y} B$ is a $\boldsymbol{\Sigma}_{\alpha}^{0}(X)$ set. Similarly, if $B$ is $\boldsymbol{\Pi}_{\alpha}^{0}(X \times Y)$, then $\forall^{* Y} B$ is $\boldsymbol{\Pi}_{\alpha}^{0}(X)$. 
2.7. Borel sets with $\Sigma_{\alpha}^{0}$ sections. Let $X$ and $Y$ be Polish spaces. For every countable nonzero ordinal $\alpha$, let $\mathcal{S}_{\alpha}^{\Sigma}$ be the set of Borel sets $B \subseteq$ $X \times Y$ such that for every $y \in Y$ the horizontal section $B^{y}$ is in $\boldsymbol{\Sigma}_{\alpha}^{0}(X)$. Let $\mathcal{T}_{0}^{\Pi}=\left\{U \times B: U \in \boldsymbol{\Sigma}_{1}^{0}(X), B \in \boldsymbol{B}(Y)\right\}$. For every countable nonzero ordinal $\alpha$, we define $\mathcal{T}_{\alpha}^{\Sigma}$ and $\mathcal{T}_{\alpha}^{\Pi}$ by the following induction:

$$
\mathcal{T}_{\alpha}^{\Sigma}=\bigvee^{\omega}\left(\bigcup_{\beta<\alpha} \mathcal{T}_{\beta}^{\Pi}\right), \quad \mathcal{T}_{\alpha}^{\Pi}=\left\{B \subseteq X \times Y:(X \times Y) \backslash B \in \mathcal{T}_{\alpha}^{\Sigma}\right\}
$$

where $\bigvee^{\omega}$ is the closure under countable unions. It is clear that $\boldsymbol{B}(X \times Y)$ $=\bigcup_{\alpha<\omega_{1}} \mathcal{T}_{\alpha}^{\Sigma}=\bigcup_{\alpha<\omega_{1}} \mathcal{T}_{\alpha}^{\Pi}$. It is also clear that $\mathcal{T}_{\alpha}^{\Sigma} \subseteq \mathcal{S}_{\alpha}^{\Sigma}$. The following proposition, a subtle result due to A. Louveau, says the converse is also true (see [16, Theorem 1, p. 375]).

Proposition 2.6. For every countable nonzero ordinal $\alpha, \mathcal{T}_{\alpha}^{\Sigma}=\mathcal{S}_{\alpha}^{\Sigma}$.

Proposition 2.6 will play an important role in Section 4. We refer to [16, $\S 3]$ for more about it.

3. Difference property of Lebesgue measurable functions and Borel functions under RVMC. In this section we prove Theorems 1.2 and 1.3. These results should be contrasted with the earlier results mentioned in the introduction showing that under $\mathrm{CH}$ none of the classes between the Baire class 2 and the Lebesgue measurable functions has the difference property.

3.1. Lemmas on measurability of integrals. In order to prove these theorems we need several lemmas asserting the preservation of the measurability of functions under integration. Our first lemma is due to Laczkovich and Miller [15, Lemma 6].

Lemma 3.1. Let $X$ be a Polish space and $(Y, \mathcal{P}(Y), \mu)$ be a universal probability measure space. Let $\alpha$ be a countable ordinal. Let $F: X \times Y \rightarrow \mathbb{R}$ be a bounded function such that for every $y \in Y$ the horizontal section $F^{y}: x \mapsto F(x, y)$ is of Baire class $\alpha$. Then the function

$$
x \mapsto \int_{Y} F(x, y) d \mu(y)
$$

is also of Baire class $\alpha$.

Lemma 3.2. Let $X$ be a Polish space and $(Y, \mathcal{P}(Y), \mu)$ be a universal probability measure space. Let $F: X \times Y \rightarrow \mathbb{R}$ be a bounded function such that for every $y \in Y$ the horizontal section $F^{y}$ is Borel. Then the function $x \mapsto \int_{Y} F(x, y) d \mu(y)$ is Borel.

Proof. For each countable ordinal $\alpha$, let $Y_{\alpha}$ be the set of $y \in Y$ for which $F^{y}$ is of Baire class $\alpha$. Then we have $Y_{\alpha} \subseteq Y_{\beta}\left(\alpha \leq \beta<\omega_{1}\right)$ and 
$Y=\bigcup_{\alpha<\omega_{1}} Y_{\alpha}$. It follows that $\mu\left(Y_{\alpha}\right) \leq \mu\left(Y_{\beta}\right)\left(\alpha \leq \beta<\omega_{1}\right)$. Since there is no strictly increasing $\omega_{1}$-sequence of real numbers, there is an $\alpha<\omega_{1}$ such that $\mu\left(Y_{\alpha}\right)=\mu\left(Y_{\beta}\right)\left(\alpha \leq \beta<\omega_{1}\right)$.

By Lemma 2.1, $\mu$ is $\omega_{2}$-additive. So we have $\mu\left(Y_{\alpha}\right)=\mu(Y)=1$. Therefore, for every $x \in X$ we have

$$
\int_{Y} F(x, y) d \mu(y)=\int_{Y_{\alpha}} F(x, y) d \mu(y) .
$$

By Lemma 3.1 for the universal probability measure space $\left(Y_{\alpha}, \mathcal{P}\left(Y_{\alpha}\right), \mu\right)$, we conclude that the function $x \mapsto \int_{Y} F(x, y) d \mu(y)$ is of Baire class $\alpha$.

Lemma 3.3. Let $X$ be a Polish space and $(Y, \mathcal{P}(Y), \mu)$ be a $\sigma$-finite universal measure space. Let $F: X \times Y \rightarrow \mathbb{R}$ be such that for every $y \in Y$ the horizontal section $F^{y}$ is Borel and for every $x \in X$ the vertical section $F_{x}$ is $\mu$-integrable. Then the function $x \mapsto \int_{Y} F(x, y) d \mu(y)$ is Borel.

Proof. Since $\mu$ is $\sigma$-finite, we can find $Y_{n} \subseteq Y$ with $\mu\left(Y_{n}\right)<\infty(n<\omega)$ such that $Y_{n} \subseteq Y_{m}$ for $n \leq m$ and $Y=\bigcup_{n<\omega} Y_{n}$.

For each positive integer $N$, let

$$
F_{N}(x, y)= \begin{cases}N & \text { if } F(x, y) \geq N \\ F(x, y) & \text { if }-N<F(x, y)<N, \\ -N & \text { if } F(x, y) \leq-N\end{cases}
$$

Then $F_{N}$ is bounded and the horizontal section $\left(F_{N}\right)^{y}$ is Borel for every $y \in Y$. Therefore by Lemma 3.2 the function

$$
f_{N}(x)=\int_{Y_{N}} F_{N}(x, y) d \mu(y)
$$

is Borel. Since the vertical section $F_{x}$ is $\mu$-integrable for every $x \in X$ we have

$$
\lim _{N \rightarrow \infty} f_{N}(x)=\lim _{N \rightarrow \infty} \int_{Y_{N}} F_{N}(x, y) d \mu(y)=\int_{Y} F(x, y) d \mu(y) .
$$

So the function $x \mapsto \int_{Y} F(x, y) d \mu(y)$ is the pointwise limit of a sequence of Borel functions, hence it is Borel, as required.

Lemma 3.4. Let $X$ be a Polish space and let $\lambda$ be a $\sigma$-finite Borel measure on $X$. Let $(Y, \mathcal{P}(Y), \mu)$ be a $\sigma$-finite universal measure space. Let $F: X \times Y \rightarrow \mathbb{R}$ be such that for every $y \in Y$ the horizontal section $F^{y}$ is $\lambda$-measurable in the usual sense and for every $x \in X$ the vertical section $F_{x}$ is $\mu$-integrable. Then the function $x \mapsto \int_{Y} F(x, y) d \mu(y)$ is $\lambda$-measurable.

Proof. By Luzin's theorem (see e.g. [11, (17.12) Theorem, p. 108]), there is a function $G: X \times Y \rightarrow \mathbb{R}$ such that for every $y \in Y$ the horizontal section $G^{y}$ is $\boldsymbol{\Sigma}_{3}^{0}$-measurable and $\left\{x: G^{y}(x) \neq F^{y}(x)\right\}$ is $\lambda$-null. Then, by Lemma 2.2. $G$ is $\boldsymbol{B}(X) \otimes \mathcal{P}(Y)$-measurable. Let $E=\{\langle x, y\rangle: G(x, y) \neq$ 
$F(x, y)\}$. Let $D \subseteq X \times Y$ be such that $D^{y}$ is a $\lambda$-null $\Pi_{2}^{0}$ set containing $E^{y}$ $(y \in Y)$. Then $D \in \boldsymbol{B}(X) \otimes \mathcal{P}(Y)$ by Lemma 2.2. So by the Fubini theorem we have

$$
\begin{aligned}
(\lambda \otimes \mu)(D) & =\iint_{X \times Y} \chi_{D}(x, y) d(\lambda \otimes \mu)(x, y) \\
& =\int_{Y}\left(\int_{X} \chi_{D}(x, y) d \lambda(x)\right) d \mu(y) \\
& =\int_{Y} \lambda\left(D^{y}\right) d \mu(y)=0 .
\end{aligned}
$$

It follows that $\mu\left(\left\{y: F_{x}(y) \neq G_{x}(y)\right\}\right)=0$ for $\lambda$-almost every $x$. So for $\lambda$-almost every $x$, the section $G_{x}$ is $\mu$-integrable and

$$
\int_{Y} F(x, y) d \mu(y)=\int_{Y} G(x, y) d \mu(y) .
$$

By Lemma 3.3 the function $x \mapsto \int_{Y} G(x, y) d \mu(y)$ is Borel. Therefore the function $x \mapsto \int_{Y} F(x, y) d \mu(y)$ is $\lambda$-measurable, as required.

3.2. Proof of Theorem 1.2, Let $f: \mathbb{R} \rightarrow \mathbb{R}$ be such that $\Delta_{h} f$ is Lebesgue measurable for every $h \in \mathbb{R}$. By a result of Laczkovich [12, Theorem 3], $f=g+\theta+S$ with a Lebesgue measurable $g$, an additive $\theta$, and a function $S$ such that for every $h \in \mathbb{R}$ the set $\left\{x: \Delta_{h} S(x) \neq 0\right\}$ is Lebesgue null. Therefore it is sufficient to show that every such function $S$ is Lebesgue measurable.

Let $\kappa$ be an atomlessly measurable cardinal. Then $\kappa \leq \mathfrak{c}$ and the Lebesgue measure $\lambda$ can be extended to a $\kappa$-additive universal measure (see [6. Theorem $1 \mathrm{D}(\mathrm{e})]$, [19], or [10, Section 2] for the details). So let $\mu$ be a $\kappa$-additive universal measure on $\mathbb{R}$ that extends the Lebesgue measure.

Let $F(x, y)=S(x+y)-S(x)-S(y)$. Then for every $y$ we have $F^{y}(x)=$ $-S(y)$ for $\lambda$-almost every $x$. Similarly, for every $x$ we have $F_{x}(y)=-S(x)$ for $\mu$-almost every $y$. In particular, $\left.F_{x}\right|_{[0,1]}$ is $\mu$-integrable for every $x \in \mathbb{R}$. By Lemma 3.4 the function

$$
x \mapsto \int_{[0,1]} F(x, y) d \mu(y)=-S(x)
$$

is $\lambda$-measurable, i.e., Lebesgue measurable. Therefore $S$ is Lebesgue measurable, as required.

3.3. Proof of Theorem 1.3. Let $f: \mathbb{R} \rightarrow \mathbb{R}$ be such that $\Delta_{h} f$ is Borel for every $h \in \mathbb{R}$. By Theorem [1.2, $f=g+\theta$ with Lebesgue measurable $g$ and additive $\theta$. Let $\varphi: \mathbb{R} \rightarrow \mathbb{R}$ be a Borel function such that $g(x)=\varphi(x)$ for almost every $x$. Let $S=g-\varphi$. Then for every $h \in \mathbb{R}, \Delta_{h} S$ is a Borel function and $\Delta_{h} S(x)=0$ for almost every $x$. 
In order to show that every such $S$ is Borel, we proceed as in the proof of Theorem 1.2 but this time we apply Lemma 3.3 rather than Lemma 3.4 .

The difference property for Baire class $\alpha$ functions follows from the difference property of Borel functions through Theorem 1.4

4. Borel functions with Baire $\alpha$ differences. This section is devoted to the proof of Theorem 1.4, which gives an affirmative answer to [12, Problem 3] of M. Laczkovich.

As we have mentioned in the Introduction, unlike the results of Section 3 which use measure theory conceptually as well as technically, the proof of Theorem 1.4 presented in this section uses only Baire category. However, at the end of this section we sketch an alternative proof which is of measure-theoretic nature. We will discuss the interplay of different proofs with possible consistent generalizations of our results in Section 5 .

We will need the following folklore lemma. For every $x \in \mathbb{R}$ and $\varepsilon>0$, let $B(x, \varepsilon)=\{y \in \mathbb{R}:|y-x|<\varepsilon\}$.

Lemma 4.1. Let $X$ be a Polish space and let $f: X \rightarrow \mathbb{R}$ be a Baire measurable function. Then there exists a comeager $\boldsymbol{\Pi}_{2}^{0}(X)$ set $G$ and a function $g: X \rightarrow \mathbb{R}$ of Baire class 1 such that $\left.f\right|_{G}=\left.g\right|_{G}$.

Proof. By Nikodým's theorem (see e.g. [11, (8.38) Theorem, p. 52]), there exists a comeager $\Pi_{2}^{0}(X)$ set $G$ such that $\left.f\right|_{G}$ is continuous. We define $h: X \rightarrow \mathbb{R} \cup\{ \pm \infty\}$ by

$$
h(x)=\lim _{\varepsilon \rightarrow+0}(\inf \{f(y): y \in B(x, \varepsilon) \cap G\}) \quad(x \in X) .
$$

By the continuity of $\left.f\right|_{G},\left.h\right|_{G}=\left.f\right|_{G}$. For every $c \in \mathbb{R}$ the set $\{x \in X: h(x)>c\}$ is open. It follows that $h$ is of Baire class 1 and the set $h^{-1}(\{ \pm \infty\})$ is $\Pi_{2}^{0}(X)$. Since $h^{-1}(\{ \pm \infty\})$ and $G$ are disjoint $\Pi_{2}^{0}$ sets, by the separation principle there exists a $\boldsymbol{\Delta}_{2}^{0}(X)$ set $D$ such that $h^{-1}(\{ \pm \infty\}) \subseteq D$ and $G \cap D=\emptyset$. We define $g: X \rightarrow \mathbb{R}$ by

$$
g(x)= \begin{cases}h(x) & \text { if } x \in X \backslash D \\ 0 & \text { if } x \in D\end{cases}
$$

Then $g$ is of Baire class 1 and $\left.g\right|_{G}=\left.f\right|_{G}$, as required.

Our main lemma is the following. For every topological space $Y$ and $A \subseteq Y, \operatorname{cl}_{Y}(A)$ denotes the closure of $A$.

Lemma 4.2. Let $X$ and $Y$ be Polish spaces. Let $\alpha$ be a countable nonzero ordinal. Let $B \subseteq X \times Y$ be a Borel set whose horizontal sections $B^{y}$ are in $\boldsymbol{\Sigma}_{\alpha}^{0}(X)$ for every $y \in Y$. Then there is a comeager $\boldsymbol{\Pi}_{2}^{0}(Y)$ set $G$ such that $B \cap(X \times G)$ is in $\Sigma_{\alpha}^{0}(X \times G)$.

Proof. First we show that for every set $A \subseteq Y$ with the Baire property, there is a comeager $\boldsymbol{\Pi}_{2}^{0}(Y)$ set $\mathcal{G}(A)$ such that $A \cap \mathcal{G}(A)$ is relatively clopen in 
$\mathcal{G}(A)$. By [11, (8.23) Proposition, p. 47], for every $A$ with the Baire property there is an open set $\mathcal{O}(A) \subseteq Y$ such that $(A \backslash \mathcal{O}(A)) \cup(\mathcal{O}(A) \backslash A)$ is meager. Let $\mathcal{E}(A)$ be a meager $\boldsymbol{\Sigma}_{2}^{0}(Y)$ set containing the meager sets $A \backslash \mathcal{O}(A)$, $\mathcal{O}(A) \backslash A$ and $\operatorname{cl}_{Y}(\mathcal{O}(A)) \backslash \mathcal{O}(A)$. Let $\mathcal{G}(A)=Y \backslash \mathcal{E}(A)$; we show that this definition fulfills the requirements.

It is immediate that $\mathcal{G}(A)$ is a comeager $\Pi_{2}^{0}(Y)$ set. From $A=(A \cap \mathcal{O}(A))$ $\cup(A \backslash \mathcal{O}(A))$ we have

$$
A \cap \mathcal{G}(A)=A \backslash \mathcal{E}(A)=A \cap \mathcal{O}(A) \backslash \mathcal{E}(A)=\mathcal{O}(A) \backslash \mathcal{E}(A)=\mathcal{O}(A) \cap \mathcal{G}(A),
$$

i.e. $A \cap \mathcal{G}(A)$ is relatively open in $\mathcal{G}(A)$. Similarly,

$$
A \cap \mathcal{G}(A)=\mathcal{O}(A) \backslash \mathcal{E}(A)=\operatorname{cl}_{Y}(\mathcal{O}(A)) \backslash \mathcal{E}(A)=\operatorname{cl}_{Y}(\mathcal{O}(A)) \cap \mathcal{G}(A),
$$

thus $A \cap \mathcal{G}(A)$ is relatively closed in $\mathcal{G}(A)$, as required.

We prove the lemma by induction on $\alpha$. For $\alpha=1, B$ is a Borel set with open sections. So by Proposition 2.6, there are open sets $U(n) \subseteq X$ and Borel sets $B(n) \subseteq Y$ such that $B=\bigcup_{n<\omega}(U(n) \times B(n))$. Let $G=$ $\bigcap_{n<\omega} \mathcal{G}(B(n))$. Since $B(n) \cap \mathcal{G}(B(n))$ is clopen in $\mathcal{G}(B(n)), B(n) \cap G$ is clopen in $G$. It follows that $(U(n) \times B(n)) \cap(X \times G)$ is open in $X \times G$. So $B \cap(X \times G)=\bigcup_{n<\omega}(U(n) \times B(n)) \cap(X \times G)$ is also open in $X \times G$. This completes the proof of the $\alpha=1$ case.

Let now $1<\alpha<\omega_{1}$ and suppose the statement holds for every $\beta<\alpha$. Let $B \subseteq X \times Y$ be a Borel set whose sections $B^{y}$ are in $\boldsymbol{\Sigma}_{\alpha}^{0}(X)$ for every $y \in Y$. By Proposition 2.6, there are Borel sets $B(n) \subseteq X \times Y$ and ordinals $\alpha_{n}<\alpha$ such that $B=\bigcup_{n<\omega} B(n)$ and for every $n<\omega$ and every $y \in Y$ the section $(B(n))^{y}$ is in $\boldsymbol{\Pi}_{\alpha_{n}}^{0}(X)$. By applying the induction hypothesis to $Y \backslash B(n)(n<\omega)$, we obtain comeager $\boldsymbol{\Pi}_{2}^{0}(Y)$ sets $G(n)$ such that $B(n) \cap$ $(X \times G(n))$ is in $\Pi_{\alpha_{n}}^{0}(X \times G(n))$. Then $G=\bigcap_{n<\omega} G(n)$ fulfills the requirements.

An important corollary of Lemma 4.2 is given by the next lemma.

Lemma 4.3. Let $X$ and $Y$ be Polish spaces and $\alpha$ be a countable nonzero ordinal. Let $B \subseteq X \times Y$ be a Borel set whose horizontal sections $B^{y}$ are in $\boldsymbol{\Sigma}_{\alpha}^{0}(X)$ for every $y \in Y$. Then $\exists^{* Y} B$ is a $\boldsymbol{\Sigma}_{\alpha}^{0}(X)$ set.

Proof. By Lemma 4.2 there is a comeager $\Pi_{2}^{0}(Y)$ set $G$ such that $B \cap$ $(X \times G)$ is in $\boldsymbol{\Sigma}_{\alpha}^{0}(X \times G)$. We have $\exists^{* Y} B=\exists^{* G}(B \cap(X \times G))$ since $G$ is comeager. By Lemma 2.5, the right hand side is a $\Sigma_{\alpha}^{0}(X)$ set, as required.

4.1. Proof of Theorem 1.4. For $\alpha=0$, i.e., for continuous functions, the result is due to de Bruijn (see [2] or [14]). So we can assume $\alpha \geq 1$.

Let $f$ satisfy the conditions of Theorem 1.4. By Lemma 4.1 there is a Baire class 1 function $g: \mathbb{R} \rightarrow \mathbb{R}$ such that $\{x \in \mathbb{R}: f(x) \neq g(x)\}$ is meager. Set $n=f-g$. Then it is enough to see that the Borel function $n$ is of Baire 
class $\alpha$. By [11, (24.3) Theorem, p. 190], it is sufficient to show that for every open set $U \subseteq \mathbb{R}$ the inverse image $n^{-1}(U)$ is in $\Sigma_{\alpha+1}^{0}(\mathbb{R})$.

We define $F: \mathbb{R} \times \mathbb{R} \rightarrow \mathbb{R}$ by $F(x, y)=-n(x+y)+n(x)+n(y)$ and set $B=F^{-1}(U)$. For every $y \in \mathbb{R}$ the section $F^{y}=n(y)-\Delta_{y} n$ is of Baire class $\alpha$. So by Lebesgue's theorem, $B^{y}=\left(F^{y}\right)^{-1}(U)$ is a $\Sigma_{\alpha+1}^{0}(\mathbb{R})$ set. Then by Lemma 4.3 the set $\exists^{* \mathbb{R}} B$ is a $\boldsymbol{\Sigma}_{\alpha+1}^{0}(\mathbb{R})$ set.

Fix an arbitrary $x \in \mathbb{R}$. By definition,

$$
B_{x}=\{y \in \mathbb{R}:-n(x+y)+n(x)+n(y) \in U\} .
$$

Since both $\{y: n(x+y)=0\}$ and $\{y: n(y)=0\}$ are comeager, $B_{x}$ is nonmeager if and only if $n(x) \in U$. Hence $n^{-1}(U)=\exists^{* \mathbb{R}} B$, which is a $\Sigma_{\alpha+1}^{0}(\mathbb{R})$ set. This completes the proof.

To conclude this section, let us briefly indicate how Theorem 1.4 can be proved using a measure-theoretic approach. The counterpart of Lemma 4.1 is given by the following result, which follows by a straightforward application of Luzin's theorem (see e.g. [11, (17.12) Theorem, p. 108]).

Lemma 4.4. Let $(X, \tau)$ be a Polish space and let $\mu$ be a Borel measure on $X$ satisfying $\mu(B(x, r))<\infty$ for every $x \in X$ and $r>0$. Let $f: X \rightarrow \mathbb{R}$ be a $\mu$-measurable function. Then for every $\varepsilon>0$ there is a closed set $F \subseteq X$ and a continuous function $g: X \rightarrow \mathbb{R}$ such that $\mu(X \backslash F)<\varepsilon$ and $\left.f\right|_{F}=\left.g\right|_{F}$.

We note that it is not enough to assume that $\mu$ is $\sigma$-finite, and we cannot achieve $\mu(X \backslash F)=0$ even if we relax the condition on $g$ to be merely of Baire class 1.

The following result is the counterpart of Lemma 4.2 .

Lemma 4.5. Let $X$ and $Y$ be Polish spaces and let $\mu$ be a Borel measure on $Y$ satisfying $\mu(B(y, r))<\infty(y \in Y, r>0)$. Let $\alpha$ be a countable nonzero ordinal. Let $B \subseteq X \times Y$ be a Borel set whose horizontal sections $B^{y}$ are in $\boldsymbol{\Sigma}_{\alpha}^{0}(X)$ for every $y \in Y$. Then for every $\varepsilon>0$ there is a closed set $F \subseteq Y$ such that $\mu(Y \backslash F)<\varepsilon$ and $B \cap(X \times F)$ is in $\boldsymbol{\Sigma}_{\alpha}^{0}(X \times F)$.

Proof. By [11, (17.11) Theorem, p. 107], for every Borel set $A \subseteq Y$ we have $\mu(A)=\sup \{\mu(K): K \subseteq A, K$ closed $\}$. So for every Borel set $A \subseteq Y$ and every $\varepsilon>0$ there is a closed set $\mathcal{G}(A) \subseteq Y$ such that $\mu(Y \backslash \mathcal{G}(A))<\varepsilon$ and $A \cap \mathcal{G}(A)$ is clopen in $\mathcal{G}(A)$. Then the rest of the proof is an inductive argument, as for Lemma 4.2 .

The corollary of Lemma 4.5, analogous to Lemma 4.3, follows from a result of Montgomery (see e.g. [11, (22.25) Exercise, p. 175]).

Lemma 4.6. Let $X$ and $Y$ be Polish spaces and let $\mu$ be a Borel measure on $Y$ satisfying $\mu(B(y, r))<\infty(y \in Y, r>0)$. Let $\alpha$ be a countable nonzero ordinal and let $B \subseteq X \times Y$ be a Borel set whose horizontal sections $B^{y}$ are 
in $\boldsymbol{\Sigma}_{\alpha}^{0}(X)$ for every $y \in Y$. Then for every $\varepsilon \geq 0,\left\{x \in X: \mu\left(B_{x}\right)>\varepsilon\right\}$ is a $\boldsymbol{\Sigma}_{\alpha}^{0}(X)$ set.

Using these lemmas, the proof of Theorem 1.4 using Lebesgue measurability of Borel functions follows as in the Baire category approach above.

\section{Generalizations}

5.1. Difference property of Lebesgue measurable functions. Recall that $\operatorname{cov}(\mathcal{N})$ denotes the smallest cardinality of a family of Lebesgue null sets that covers $\mathbb{R} ; \operatorname{non}(\mathcal{N})$ is the smallest cardinality of a Lebesgue nonnull set; and $\operatorname{non}^{\star}(\mathcal{N})$ is the smallest cardinality $\kappa$ such that every Lebesgue nonnull set has a Lebesgue nonnull subset of cardinality $\kappa$ (see [1] for more on cardinal invariants).

It was shown in [13] that the difference property of Lebesgue measurable functions follows from the cardinal inequality $\operatorname{non}^{\star}(\mathcal{N})<\operatorname{cov}(\mathcal{N})$. This inequality is known to hold in random real models (see [13]). If $\kappa$ is an atomlessly measurable cardinal, then we know $\operatorname{cov}(\mathcal{N}) \geq \kappa$ and $\operatorname{non}(\mathcal{N})=\omega_{1}$. But we do not know the answer to the following.

QUESTION. Is $\operatorname{non}^{\star}(\mathcal{N})<\operatorname{cov}(\mathcal{N})$ a consequence of the existence of an atomlessly measurable cardinal?

We note here that, unlike $\operatorname{non}(\mathcal{N})$, the cardinal $\operatorname{non}^{\star}(\mathcal{N})$ is not determined by the presence of an atomlessly measurable cardinal. If $\kappa$ is twovalued measurable and $\mathrm{CH}$ holds, then the forcing notion $\mathbb{B}_{\kappa}$ for adding $\kappa$ many random reals forces that $\kappa$ is atomlessly measurable and $\operatorname{non}^{\star}(\mathcal{N})$ $=\omega_{1}$. On the other hand, if $\kappa$ is two-valued measurable and $\mathrm{MA}+\neg \mathrm{CH}$ holds, then $\mathbb{B}_{\kappa}$ forces that $\kappa$ is atomlessly measurable and $\operatorname{non}^{\star}(\mathcal{N})>\omega_{1}$. However, as we have mentioned, $\operatorname{cov}(\mathcal{N})$ is forced to be not less than $\kappa$. Therefore it anyway becomes far bigger than $\operatorname{non}^{\star}(\mathcal{N})$, which does not exceed the size of the ground model's continuum. So these two cases do not provide counter-examples to our Question.

Finally, we would like to point out that Lemma 3.2 can be applied to a problem studied in [15], from which we have adopted Lemma 3.1. The following is a modified version of [15, Theorem 2].

Proposition 5.1. Suppose there exists an atomlessly measurable cardinal. Let $f: \mathbb{R}^{2} \rightarrow \mathbb{R}$ be such that the vertical sections $f_{x}$ are approximately continuous and the horizontal sections $f_{y}$ are Borel for all $x, y \in \mathbb{R}$. Then $f$ is Borel as a function of two variables.

5.2. Difference property of the Baire classes. Let us recall that by a classical result of N. G. de Bruijn, the class of continuous functions has the difference property, while by a result of M. Laczkovich, the class of 
Baire class 1 functions has the difference property (see [2] and [14]); that is, for $\alpha=0$ and $\alpha=1$ the conclusion of Theorem 1.4 holds without any definability assumption on $f$.

On the other hand, under $V=L$, there exists an analytic set $A \subseteq \mathbb{R}$ such that both $A$ and $\mathbb{R} \backslash A$ are uncountable while $(A+t) \backslash A$ is countable for every $t \in \mathbb{R}$ (see [3, Theorem 4.7]). It is known that such an analytic set $A$ is non-Borel and comeager, thus $\chi_{A}$ shows that the conclusion of Theorem 1.4 may fail for $\alpha=2$ and a $\Delta_{2}^{1}$-measurable function.

The main lemma of Theorem 1.4. Lemma 4.2, is also optimal in the following sense.

Proposition 5.2. Assume $V=L$. Then there is a function $f: \mathbb{R} \rightarrow \mathbb{R}$ with a coanalytic graph such that $\operatorname{Graph}(f) \cap(B \times \mathbb{R})$ is not Borel for every set $B$ which is either nonnull or nonmeager.

Proof. It is well known that under $V=L$ there exists a $\Delta_{2}^{1}$ set which is not Lebesgue measurable in any nonnull Borel set and does not have the Baire property in any nonmeager Borel set; e.g., the well-ordering $<_{L} \cap$ $\left({ }^{\omega} \omega \times{ }^{\omega} \omega\right)$ (see e.g. [9, Corollary 25.28, p. 495]). So let $P \subseteq \mathbb{R}$ be such a $\boldsymbol{\Delta}_{2}^{1}$ set. Then both $P$ and $\mathbb{R} \backslash P$ are projections of $\boldsymbol{\Pi}_{1}^{1}$ sets, i.e. there are $\Pi_{1}^{1}$ sets $A_{1} \subseteq \mathbb{R} \times[0,1)$ and $A_{2} \subseteq \mathbb{R} \times[1,2)$ such that $P=\operatorname{proj}_{\mathbb{R}}\left(A_{1}\right)$ and $\mathbb{R} \backslash P=\operatorname{proj}_{\mathbb{R}}\left(A_{2}\right)$.

Let $A=A_{1} \cup A_{2}$. By [11, (36.15) Theorem, p. 306], $A$ can be uniformized by a $\Pi_{1}^{1}$ set, so we can assume $A$ is the graph of a function $f: \mathbb{R} \rightarrow[0,2)$. Therefore for every $x \in \mathbb{R}$ the vertical section $A_{x}$ consists of a unique point, in particular it is closed.

Let $B \subseteq \mathbb{R}$ be a set such that $A \cap(B \times[0,2))$ is Borel. Then $P_{1}=$ $\operatorname{proj}_{\mathbb{R}}(A \cap(B \times[0,1)))$ and $P_{2}=\operatorname{proj}_{\mathbb{R}}(A \cap(B \times[1,2)))$ form a disjoint pair of analytic sets such that $P_{1} \cup P_{2}=B$ and $B \cap P=P_{1}$; in particular $B$ and $B \cap P$ are both analytic. So if $B$ is nonnull then there is a nonnull Borel set $B^{\prime} \subseteq B$ such that $B^{\prime} \cap P$ is Lebesgue measurable, a contradiction. Similarly, if $B$ is nonmeager then there is a nonmeager Borel set $B^{\prime} \subseteq B$ such that $B^{\prime} \cap P$ has the Baire property, again a contradiction. This completes the proof.

For the sake of completeness, we point out that for $\alpha=1$, Lemma 4.2 holds for coanalytic sets.

Proposition 5.3. Let $X$ and $Y$ be Polish spaces and let $A \subseteq X \times Y$ be a coanalytic set. Suppose that for every $y \in Y$ the horizontal section $A^{y}$ is open. Then there exists a comeager $\Pi_{2}^{0}(Y)$ set $G$ such that $A \cap(X \times G)$ is open in $X \times G$.

Proof. For each basic open set $I$ from a fixed countable open basis of $X$, put $B_{I}=\left\{y \in Y: I \subseteq A^{y}\right\}$. It is routine to check that $B_{I}$ is coanalytic, in 
particular it has the Baire property. Then we can proceed as in the proof of Lemma 4.2 ,

Finally, we would like to show that, e.g. under sufficient determinacy assumptions, Theorem 1.4 holds for functions satisfying weaker measurability assumptions, as follows. We call a pointclass $\boldsymbol{\Gamma}$ adequate if it is an algebra which contains the Borel sets and it is closed under taking Cartesian products.

TheOREM 5.4. Let $\boldsymbol{\Gamma}$ be an adequate pointclass such that the complements of projections of sets in $\boldsymbol{\Gamma}$ can be uniformized by Baire measurable functions. Let $f: \mathbb{R} \rightarrow \mathbb{R}$ be a $\boldsymbol{\Gamma}$-measurable function and let $\alpha$ be a countable ordinal. Suppose that for every $h \in \mathbb{R}$ the difference function $\Delta_{h} f$ is of Baire class $\alpha$. Then $f$ itself is of Baire class $\alpha$.

The assumption of Theorem 5.4 holds for $\boldsymbol{\Gamma}=\boldsymbol{\Delta}_{n}^{1}(n<\omega)$ under Projective Determinacy (see e.g. [11, Chapter 39, p. 327] or [9, Chapter 33, p. 627]). Below we only state and prove the key ingredient of its proof, namely a suitable generalization of Lemma 4.2 , then the statement follows as for Borel measurable functions.

Lemma 5.5. Let $X$ and $Y$ be Polish spaces and let $\boldsymbol{\Gamma}$ be as in Theorem 5.4. Let $\alpha$ be a countable nonzero ordinal. Let $B \subseteq X \times Y$ be in $\boldsymbol{\Gamma}$ such that the horizontal section $B^{y}$ is in $\boldsymbol{\Sigma}_{\alpha}^{0}(X)$ for every $y \in Y$. Then there is a comeager $\boldsymbol{\Pi}_{2}^{0}(Y)$ set $G$ such that $B \cap(X \times G)$ is in $\boldsymbol{\Sigma}_{\alpha}^{0}(X \times G)$.

Proof. Let $U \subseteq 2^{\omega} \times X$ be a universal $\Sigma_{\alpha}^{0}$ set, i.e. such that for every $A \in \Sigma_{\alpha}^{0}(X)$ there is a $t \in 2^{\omega}$ with $U_{t}=A$. Let

$$
\begin{aligned}
P=\{(y, t, x) \in & Y \times 2^{\omega} \times X: \\
& ((x, y) \in B \wedge(t, x) \notin U) \vee((x, y) \notin B \wedge(t, x) \in U)\} .
\end{aligned}
$$

Since $\boldsymbol{\Gamma}$ is an algebra which contains the Borel sets and is closed under taking Cartesian products, we have $P \in \boldsymbol{\Gamma}\left(Y \times 2^{\omega} \times X\right)$.

Note that $(y, t) \in \operatorname{proj}_{Y \times 2^{\omega}}(P)$ if and only if $B^{y} \neq U_{t}$. So

$$
\left\{(y, t) \in Y \times 2^{\omega}: B^{y}=U_{t}\right\}=\left(Y \times 2^{\omega}\right) \backslash \operatorname{proj}_{Y \times 2^{\omega}}(P) .
$$

By our assumption on $B$, for every $y \in Y$ there exists a $t \in 2^{\omega}$ such that $B^{y}=U_{t}$. By our assumption on $\boldsymbol{\Gamma}$, there is a Baire measurable uniformizing function of the set in (1), i.e. a Baire measurable function $f: Y \rightarrow 2^{\omega}$ such that $B^{y}=U_{f(y)}(y \in Y)$.

By Nikodým's theorem (see e.g. [11, (8.38) Theorem, p. 52]), there is a comeager $\Pi_{2}^{0}(Y)$ set $G$ such that $\left.f\right|_{G}: G \rightarrow 2^{\omega}$ is continuous. We have $(x, y) \in B$ if and only if $(f(y), x) \in U$, so

$$
B \cap(X \times G)=\left\{(x, y) \in X \times G:\left(\left(\left.f\right|_{G}\right)(y), x\right) \in U\right\} .
$$

Since $\left.f\right|_{G}$ is continuous, this shows $B \cap(X \times G)$ is $\Sigma_{\alpha}^{0}(X \times G)$, as required. 
A similar generalization is possible for Lemma 4.5 in the approach using Lebesgue measure. However, we are far from understanding the precise consistency strength of the difference property of the class of Borel measurable functions or of the class of Baire class $\alpha$ functions. So we do not present here any further analysis of the proofs.

Acknowledgements. Research of T. Mátrai was partially supported by the OTKA grants K 61600, K 49786 and K 72655.

\section{References}

[1] T. Bartoszyński and H. Judah, Set Theory. On the Structure of the Real Line, A K Peters, Wellesley, MA, 1995.

[2] N. G. de Bruijn, Functions whose differences belong to a given class, Nieuw Arch. Wisk. 23 (1951), 194-218.

[3] J. Cichoń, A. Jasiński, A. Kamburelis, and R. L. Szczepaniak, On translations of subsets of the real line, Proc. Amer. Math. Soc. 130 (2001), 1833-1842.

[4] K. Ciesielski and J. Pawlikowski, The Covering Property Axiom CPA, Cambridge Univ. Press, 2004.

[5] R. Filipów and I. Recław, On the difference property of Borel measurable and $(s)$ measurable functions, Acta Math. Hungar. 96 (1992), 21-25.

[6] D. Fremlin, Real-valued measurable cardinals, in: Set Theory of the Reals, H. Judah (ed.), Israel Math. Conf. Proc. 6, Bar-Ilan Univ., 1993, 151-304; http://www.essex. ac.uk/maths/staff/fremlin/index.shtm.

[7] H. Fujita, Remarks on two poblems by M. Laczkovich on functions with Borel measurable differences, Acta Math. Hungar. 117 (2007), 153-160.

[8] P. Halmos, Measure Theory, van Nostrand, 1950; 2nd ed., Springer, 2006.

[9] T. Jech, Set Theory, 3rd ed., Springer, 2003.

[10] A. Kanamori, The Higher Infinite, 2nd ed., Springer, 2003.

[11] A. S. Kechris, Classical Descriptive Set Theory, Springer, 1994.

[12] M. Laczkovich, Functions with measurable differences, Acta Math. Acad. Sci. Hungar. 35 (1980), 217-237.

[13] -, Two constructions of Sierpiński and some cardinal invariants of ideals, Real Anal. Exchange 24 (1998), 663-676.

[14] —, The difference property, in: Paul Erdős and His Mathematics (G. Halász et al., eds.), Volume I, Springer, Budapest, 2002, 363-410.

[15] M. Laczkovich and A. W. Miller, Measurability of functions with approximately continuous vertical sections and measurable horizontal sections, Colloq. Math. 69 (1995), 299-308.

[16] A. Louveau, A separation theorem for $\Sigma_{1}^{1}$, Trans. Amer. Math. Soc. 260 (1980), no. $2,363-378$.

[17] M. M. Rao, Measure Theory and Integration, Pure Appl. Math., Wiley, New York, 1987.

[18] W. Rudin, Real and Complex Analysis, 3rd ed., McGraw-Hill, Singapore, 1987.

[19] R. M. Solovay, Real-valued measurable cardinals, in: Axiomatic Set Theory, Proc. Sympos. Pure Math. 13, Part I, D. S. Scott (ed.), Amer. Math. Soc., 1971, 397-428.

[20] M. E. Taylor, Measure Theory and Integration, Grad. Stud. Math. 76, Amer. Math. Soc., Providence, 2006. 
[21] J. Yeh, Real Analysis-Theory of Measure and Integration, 2nd ed., World Sci., Singapore, 2006.

Hiroshi Fujita

Graduate School of Science and Technology

Ehime University

Matsuyama 790-8577, Japan

E-mail: fujita@math.sci.ehime-u.ac.jp
Tamás Mátrai Alfréd Rényi Institute of Mathematics Hungarian Academy of Sciences

Reáltanoda utca 13-15 H-1053 Budapest, Hungary E-mail: matrait@renyi.hu

Received 21 August 2008;

in revised form 25 November 2009 